This document is the accepted manuscript version of the following article:

Campanini, M., Trassin, M., Ederer, C., Erni, R., \& Rosse11, M. D. (2019). Buried in-plane

ferroelectric domains in fe-doped single-crystalline aurivillius thin films. ACS Applied

Electronic Materials, 1(6), 1019-1028. https://doi.org/10.1021/acsaelm.9b00180

\title{
Buried in-plane ferroelectric domains in Fe-doped single-crystalline Aurivillius thin films
}

\author{
Marco Campanini†*, Morgan Trassin $\S$, Claude Ederer†, Rolf Erni†, Marta D. Rossell†. \\ $\dagger$ †lectron Microscopy Center, Empa, Swiss Federal Laboratories for Materials Science and Technology, 8600 Dübendorf, \\ Switzerland, §Laboratory for Multifunctional Ferroic Materials, ETHZ, 8093 Zürich, Switzerland, \$Materials Theory, \\ ETHZ, 8093 Zürich, Switzerland
}

KEYWORDS Aurivillius thin films, layered ferroelectric, buried domains, doping, differential-phase contrast STEM

\begin{abstract}
Aurivillius phases constitute a promising class of materials displaying excellent ferroelectric properties, which make them fascinating potential candidates for ferroelectric-based devices. In this perspective, however, the realization of Aurivillius thin films still represents an open challenge. In this work, high-quality single-crystalline $\mathrm{Bi}_{5} \mathrm{FeTi}_{3} \mathrm{O}_{15}$ Aurivillius $(m=4)$ thin films are grown by pulsed laser deposition. Their structural and polar properties are elucidated by combining several atomic-resolution scanning transmission electron microscopy (STEM) techniques. Our results prove that epitaxial strain is released by the formation of out-of-phase boundaries and the Fe dopants are distributed with a preferential mixed configuration - i.e. occupying one inner and one outer site of the 4 perovskite blocks. The results demonstrate that out-of-phase boundaries are not detrimental for the local ferroelectric polarization, whose value is in agreement with the one predicted by theory. By means of differential-phase contrast (DPC-)STEM and atomic resolution displacement mapping, we demonstrate the presence of buried in-plane ferroelectric domains with the domain walls localized at the $\mathrm{Bi}_{2} \mathrm{O}_{2}$ layers. In particular, our results demonstrate that DPC-STEM enables to map buried polar domains not accessible by surface techniques.
\end{abstract}

\section{INTRODUCTION}

Designing materials with novel properties - e.g. by epitaxial strain or by doping - has been one of the main targets of recent research regarding ferroelectric materials. In particular, strain has been demonstrated to strongly promote ferroelectric phases with very high polarization and enhanced ordering temperatures, ${ }^{1-5}$ whereas doping has been widely employed to incorporate magnetic impurities. ${ }^{6-9}$

In the last years, layered oxides have attracted continuously growing attention for being a promising playground for the design of new multiferroic materials. ${ }^{10,11}$ The structure of the layered oxides is typically composed by an alternation of perovskite blocks and spacing layers having a different structure, e.g. rock salt ${ }^{12}$ or fluorite ${ }^{13}$, thus forming a superstructure. The superstructure period can be easily modified by varying the number of perovskite-type unit cells stacked within the perovskite blocks, while the spacing layer introduces an additional structural constituent that can act as donor dopant and control internal microscopic processes related to fatigue. ${ }^{14}$

Among the different layered oxides, the Aurivillius phases are particularly interesting. ${ }^{15-20}$ This family of compounds displays a layered-perovskite structure constituted by $\mathrm{m}$ perovskite blocks $\left(\mathrm{A}_{\mathrm{m}-1} \mathrm{~B}_{\mathrm{m}} \mathrm{O}_{3 \mathrm{~m}+1}\right)^{2-}$ alternating with fluorite-like layers $\left(\mathrm{Bi}_{2} \mathrm{O}_{2}\right)^{2+}$, with an overall composition given by $\mathrm{Bi}_{2} \mathrm{~A}_{\mathrm{m}}$ ${ }_{1} \mathrm{~B}_{\mathrm{m}} \mathrm{O}_{3 \mathrm{~m}+3}$. These compounds have shown excellent ferroelectric properties, i.e. high Curie temperatures, strong saturation polarization and low fatigue. ${ }^{14,21}$

Incorporating magnetic dopants in the Aurivillius phases represents a potential route to induce magnetic properties to a performant ferroelectric material, thus creating a new multiferroic. Preliminary $a b$ initio calculations ${ }^{22,23}$ predicted that the epitaxial strain could play an important role in determining the distribution of magnetic dopants in the Aurivillius phase. Although in the last decade Aurivillius compounds exhibiting multiferroic properties have been reported - typically in the form of polycrystalline samples ${ }^{9,17,20,24-30}$ - the accurate description of the distribution of the magnetic ions at the base of the magnetic ordering has not yet been completely addressed in such an intricate system. Additionally, a detailed experimental investigation of the atomic-scale structural distortions responsible for the ferroelectric properties of the Aurivillius phase is still missing.

A significant step forward in the understanding of the fundamental properties of strained and doped Aurivillius systems could be achieved by growing high-quality singlecrystalline epitaxial thin films. The realization of singlecrystalline films is crucial for relating the strain state of the Aurivillius phase to its ferroelectric and magnetic properties, not achievable in polycrystalline specimens. Despite previous works reported about the epitaxial growth of polycrystalline Aurivillius thin films on Si substrates by $\mathrm{PLD}^{31,32}$ and $\mathrm{CVD}^{33}$, the realization of single-crystalline Aurivillius thin films has remained until now an open challenge. Moreover, the possibility of controlling the ordering of the magnetic ions by a proper choice of the substrate further raises the interest for the study of single-crystalline Aurivillius films.

In this work we report the realization of high-quality singlecrystalline $\mathrm{Bi}_{5} \mathrm{FeTi}_{3} \mathrm{O}_{15}$ Aurivillius thin films grown on $\mathrm{NdGaO}_{3}$ substrates. A detailed investigation of the structural and polar properties of the films is performed by a 
combination of different scanning transmission electron microscopy techniques. Additionally, an analysis of the $\mathrm{Fe}$ dopant distribution is provided and compared with previous predictions based on density functional theory (DFT) calculations.

\section{MATERIALS AND METHODS}

\section{Thin Films Growth}

The $\mathrm{Bi}_{5} \mathrm{FeTi}_{3} \mathrm{O}_{15}$ Aurivillius thin films were epitaxially grown by pulsed laser deposition (PLD) on lattice matching $\mathrm{NdGaO}_{3}$ (001)-oriented substrates. For the optimal growth conditions, the orthorhombic NGO substrates (Crystec GmbH) were kept at $650{ }^{\circ} \mathrm{C}$ during the film deposition. The oxygen partial pressure was set to $1.5 \times 10^{-2}$ mbar. The $\mathrm{KrF}$ excimer laser intensity was set to $1 \mathrm{~J} \mathrm{~cm}^{-2}$ at a repetition rate of $8 \mathrm{~Hz}$. Samples were cooled down in the growth atmosphere.

\section{X-ray Diffraction and Atomic Force Microscopy}

The film orientation, crystal quality, strain state, and thickness were studied by X-ray diffraction (XRD) and reflectometry using a X Pert'Pro MRD (Panalytical) diffractometer equipped with a parallel-beam optics and a four-bounce $\mathrm{Ge} 220$ monochromator producing $\mathrm{Cu}-\mathrm{K} \alpha 1$ radiation at $1.5406 \AA$. The thin films surface topography was investigated by atomic force microscopy (AFM) in contact mode, using a Bruker Multimode 8.

\section{Scanning Transmission Electron Microscopy}

Scanning Transmission Electron Microscopy (STEM) was performed on an analytical FEI Titan Themis, operated at $300 \mathrm{kV}$ and equipped with an aberration corrector for the probe and Super-X Energy-Dispersive X-ray (EDX) technology. The experiments were performed by setting the beam semiconvergence angle to $18 \mathrm{mrad}$ and collecting semi-angles of 9$17 \mathrm{mrad}$ and 70-190 mrad for the annular bright field (ABF) and high-angle annular dark field (HAADF) STEM detectors, respectively. The HAADF-STEM signal is proportional to $\sim Z^{1.7}$, thus allowing for atomic-number $(Z)$ sensitive imaging and a compositional analysis of the heavy atomic columns. On the contrary, light elements - like oxygen - can be visualized by ABF-STEM, whose signal is proportional to $\sim Z^{1 / 3} \cdot{ }^{34}$ EDX maps were recorded with a beam current of $200 \mathrm{pA}$ and a dwell time of $10 \mu \mathrm{s} /$ pixel. Differential-Phase Contrast (DPC) STEM experiments were performed by means of a 4-quadrant (named: A, B, C, D) segmented annular dark field (ADF) detector, setting the collecting semi-angle to $9-57 \mathrm{mrad}$. In the medium-resolution DPC-STEM technique, the deflection of the beam due to electric fields in the specimen plane is detected as a variation of the beam intensity impinging on opposite pairs of segments of the segmented annular detector. ${ }^{35-37}$ In this way, the differential signals (A-C and BD) can be directly related to the electric fields in the specimen and, hence, to the polarization within the ferroelectric material.

\section{Image Simulations}

The HAADF- and ABF-STEM images simulation was performed using STEM_CELL ${ }^{38}$ software, setting the probe parameters and the collection angles for the detectors as they were in the experimental setup. In the simulations the specimen thickness was set to $20 \mathrm{~nm}$ and the simulated signals were obtained as the average of 15 frozen-phonon configurations.

Image Processing and Data Analysis
The HAADF-STEM images used for the strain analysis and polarization mapping were obtained as average signals of time-series of 20 frames, after rigid and non-rigid registrations performed using Smart Align software. ${ }^{39}$ The time-averaged signals were de-noised using a custom-developed non-linear algorithm based on the method proposed by $\mathrm{H}$. Du et al. ${ }^{40}$ The atomic scale polarization mapping and the quantitative estimation of the tetragonal distortion of the perovskite blocks were carried out by precisely fitting the position of the atomic columns in the HAADF- and ABF-STEM images. The fitting was performed by using 7-parameter two-dimensional gaussians in order to obtain the columns position with picometer precision. ${ }^{41}$ The EDX maps for $\mathrm{Bi}$, Ti and Fe were obtained as time-averages of 200 frames, by integrating the $\mathrm{Bi}-\mathrm{M}, \mathrm{Ti}-\mathrm{K}$ and $\mathrm{Fe}-\mathrm{K}$ edges intensities, respectively.
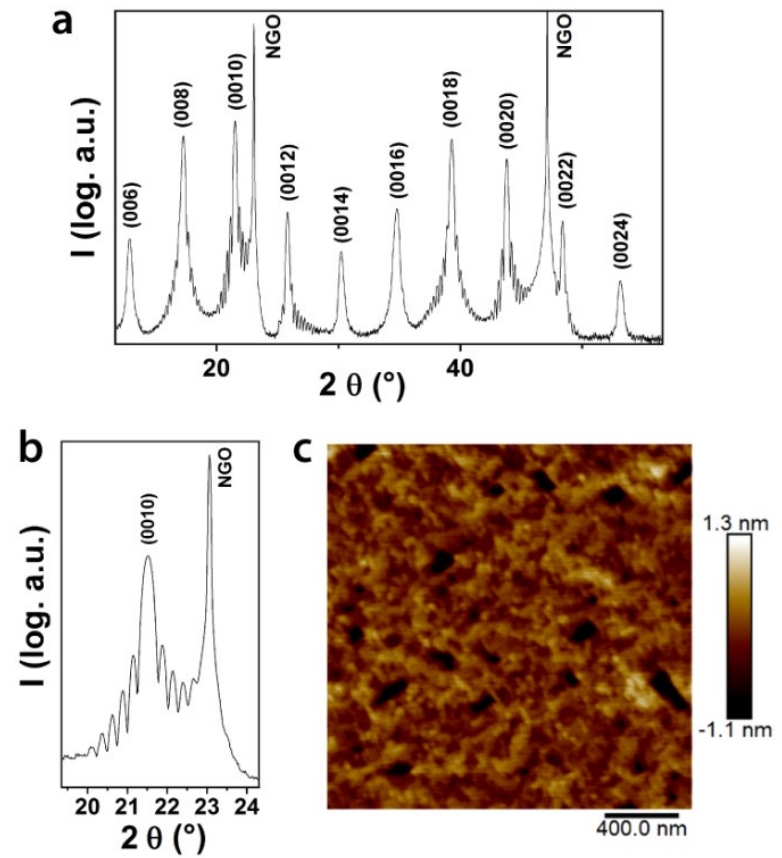

Figure 1. (a) XRD pattern of the $\mathrm{Bi}_{5} \mathrm{FeTi}_{3} \mathrm{O}_{15}$ Aurivillius thin film grown on a $\mathrm{NdGaO}_{3}$ (001)-oriented substrate. The diffraction spots correspond to the $m=4$ structure. (b) Magnified portion of the XRD pattern showing Kiessig thickness fringes. (c) AFM image showing the flat surface (roughness $\sim 1 \AA$ ) of the singlecrystalline Aurivillius thin film.

\section{RESULTS AND DISCUSSION}

High-quality single-crystalline $\mathrm{Bi}_{5} \mathrm{FeTi}_{3} \mathrm{O}_{15}$ (BFTO) thin films were grown on $\mathrm{NdGaO}_{3}$ (NGO) (001)-oriented substrate by pulsed laser deposition (PLD). The crystallographic properties of the specimens were analyzed by X-ray diffraction (XRD) to investigate the crystallinity and verify the epitaxial relationship between the film and the substrate.

Figure 1a shows a $(\theta-2 \theta)$ scan where the BFTO peaks are indexed within the $A 2{ }_{1}$ am space group, which was previously reported to describe the ferroelectric phase of the layered Aurivillius structure. ${ }^{42}$ The sole presence of $(00 d)$ peaks demonstrate that the film grows completely c-oriented and the single Aurivillius phase univocally corresponds to the $m=4$ member of the homologous series, as proven by the periodicity of the very sharp reflections. A close up diffractogram around 
the BFTO (0010) reflection in Figure $1 \mathrm{~b}$ identifies the presence of Kiessig thickness fringes and reveals the high interface quality of the film at a macroscopic scale. The atomic force microscopy (AFM) measurements confirm the low roughness expected from the XRD spectra. Figure 1c shows an AFM image over an area of $2 \times 2 \mu \mathrm{m}^{2}$ of a $34-\mathrm{nm}$-thick BFTO film. The measured roughness is in the range of $1 \AA$.

Figure 2a displays a HAADF-STEM cross-sectional image of a portion of a $40 \mathrm{~nm}$ thick BFTO thin film on the NGO substrate. The film shows defects at the interface with the substrate and out-of-phase boundaries (OPBs) distributed over the film thickness. As both BFTO and NGO have an orthorhombic structure, BFTO grows with preferential inplane crystal orientation on $\mathrm{NGO}(001)$, thus resulting in anisotropic lattice mismatch along the two in-plane BFTO directions, i.e. [100] and [010] directions. The epitaxial relationships between the c-oriented BFTO films on $\mathrm{NGO}(001)$ substrate are: $[100]_{\mathrm{BFTO}} / /[010]_{\mathrm{NGO}}$ and $[010]_{\mathrm{BFTO}} / /$ $[100]_{\mathrm{NGO}}$, with nominal misfit strains of $+0.5 \%$ (tensile) and $0.2 \%$ (compressive), respectively. Such strain values are indeed very small and cannot be detected by conventional transmission electron microscopy strain mapping methods, like, e.g., with geometric phase analysis (GPA) (see Figure S1, Supporting Information). On the other hand, it has been demonstrated that in thin films the occurrence of OPBs represents an effective mechanism - preferred over the formation of misfit dislocations - for the relief of small strains that may originate from stoichiometry defects and coalescence of single-crystalline regions. ${ }^{43}$ The presence of OPBs has indeed been formerly reported in strongly-doped Aurivillius systems. ${ }^{18}$

Despite the geometry of the layered structure is locally altered at the OPBs with small regions showing 3- or 5-unit cells in the perovskite blocks, the Aurivillius thin film globally shows the $m=4$ phase, as proven by the power spectrum (Figure 2b) of the high-resolution HAADF-STEM image. In particular, taking a profile along the [001] direction (Figure 2c) the expected peaks typical of the $A 2{ }_{1} a m$ symmetry are observed.

In order to increase the signal-to-noise ratio and perform quantitative analysis, statistically-averaged HAADF- and ABF-STEM signals were obtained in both the (110) and (100) projections. The results of the statistical analysis for HAADFand ABF-STEM signals are shown in Figure $2 \mathrm{~d}$ and $2 \mathrm{e}$, respectively. The in-phase tilting of the $\mathrm{O}$ octahedra is only visible in the ABF signal along the [100] zone axis given in Fig $2 \mathrm{e}$, in agreement with the $A 2{ }_{1} a m$ symmetry (partially sketched overlaid to the image). Nevertheless, no information about the Fe dopant distribution on the B positions (marked by light-blue dots) of the perovskite cells can be determined from the analysis of the HAADF-STEM intensity, as shown in the line-profiles given in Figure 2e and d. The low sensitivity of the HAADF-STEM signal to the substitutional dopant distribution can be attributed to (i) the rather small difference between the atomic numbers of $\mathrm{Ti}(Z=22)$ and $\mathrm{Fe}(Z=26)$, (ii) the intrinsic distortion of the outer perovskite cells of the Aurivillius structure, (iii) the averaging of the HAADF-STEM signal along the specimen thickness.
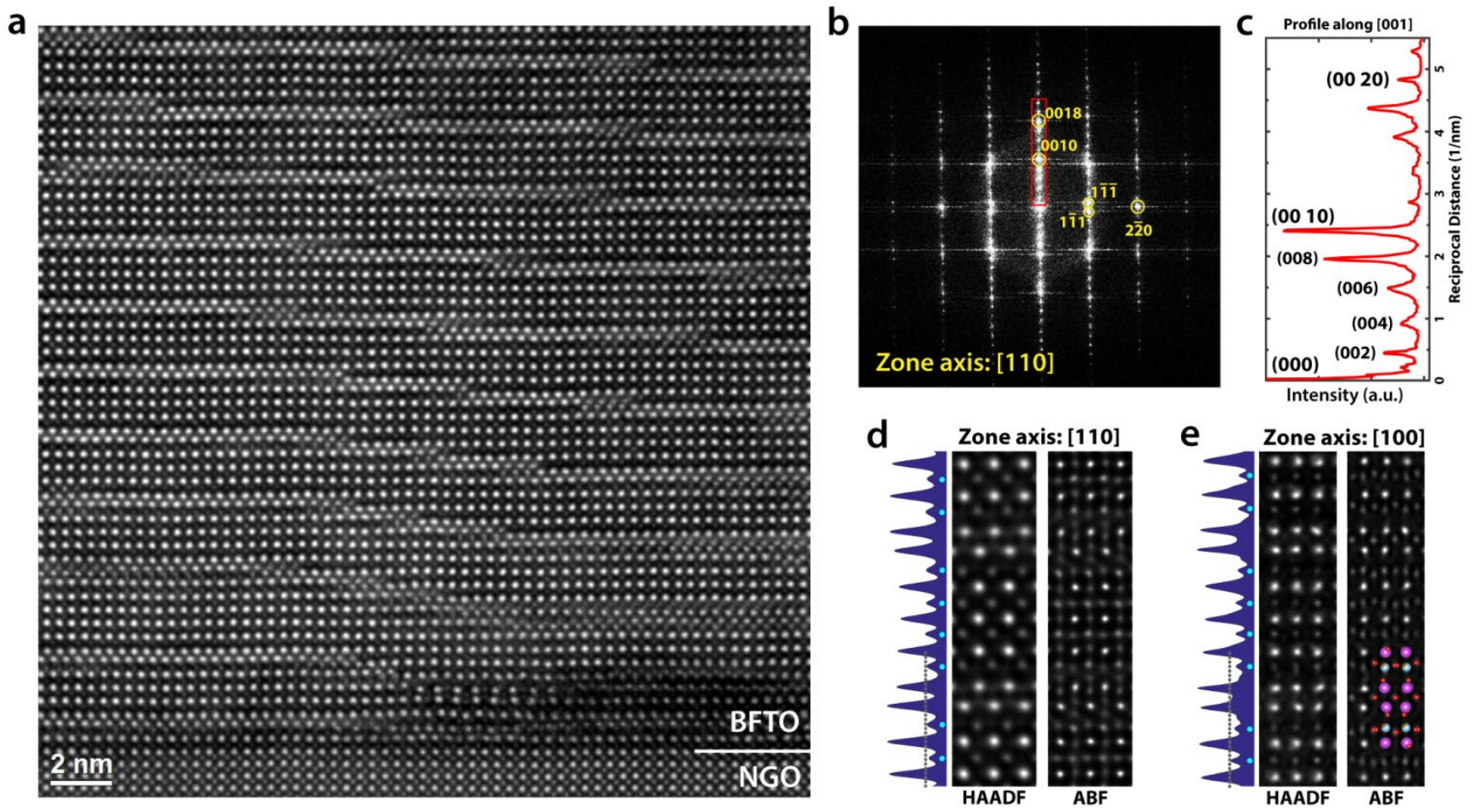

Figure 2. (a) High-resolution HAADF-STEM image of a BFTO Aurivillius thin film on a NGO substrate. Out-of-phase boundaries appear along the growing direction of the film (c-axis). (b) Corresponding power spectrum and (c) intensity profile extracted along the c-axis. (d, e) Statistically averaged portions of the HAADF- and inverted ABF-STEM signals along the [110] and [100] zone axes. The intensity profiles of the HAADF signals are given. A portion of the $A 2{ }_{1} a m$ structure (generated using VESTA ${ }^{44}$ ) is superimposed to the ABF image along the [100] zone axis to highlight the agreement between the experiments and the structural model. 


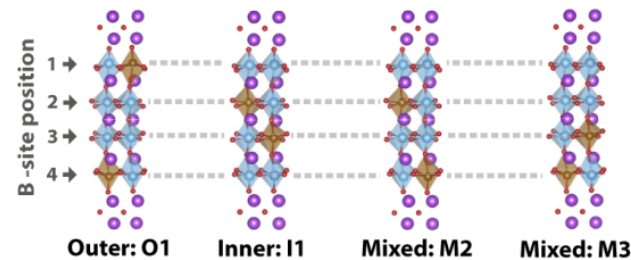

b

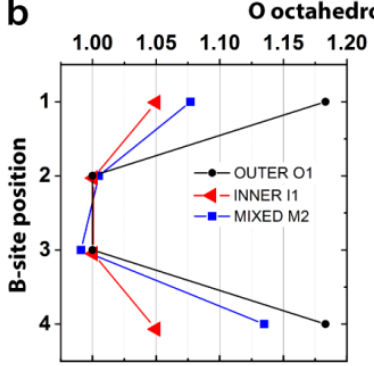

O octahedron tetragonality

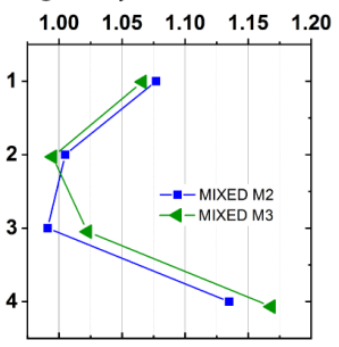

Figure 3. (a) Four selected configurations illustrative of the groups "outer", "mixed" and "inner", where the two Fe dopants occupy respectively two, one or none of the perovskite unit cells adjacent to the $\mathrm{Bi}_{2} \mathrm{O}_{2}$ layers. (b) Profiles of the average $\mathrm{O}$ octahedron tetragonality $\left(\mathrm{O}_{\perp} / \mathrm{O}_{/ /}\right)$for the given configurations. The I1 (inner) and the $\mathrm{O} 1$ (outer) configurations differ in the tetragonality of the outer sites (sites 1 and 4), while the profiles of the mixed configurations allow the identification of the $\mathrm{Fe}$ dopants sites.

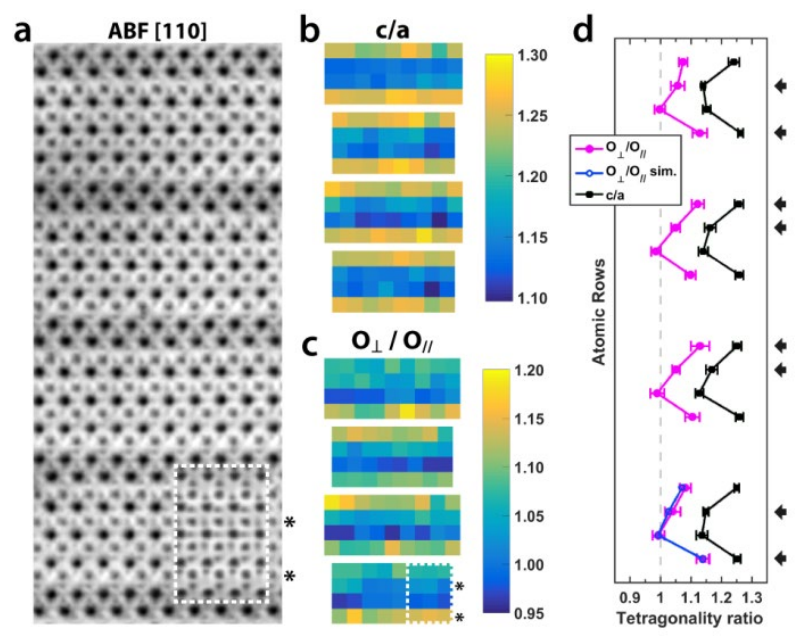

Figure 4. (a) High-resolution ABF-STEM image showing a OPBs-free portion of the sample. (b, c) Maps of the tetragonality (c/a) of the perovskite unit cell and of the octahedral site $\left(\mathrm{O}_{\perp} / \mathrm{O}_{/ /}\right)$, as obtained from PP analysis. (d) Profiles of the tetragonal distortions along the growing direction. The simulated ABF signal generated using the M2 configuration is superimposed to (a) and (c) inside the white dashed box. The layers where the Fe dopants are localized in the M2 model are marked by asterisks on the right side of the image. The preferential positions of the Fe dopants in the imaged portion of the sample are marked with black arrows.

For the $\mathrm{Bi}_{5} \mathrm{FeTi}_{3} \mathrm{O}_{15}$ Aurivillius structure with $m=4$, there are ten possible symmetrically-inequivalent configurations with different distributions of the two $\mathrm{Fe}$ and six Ti cations over the eight octahedral B sites. As pointed out by Birenbaum et al. ${ }^{22}$, a fingerprint of the dopant distribution is given by the structural distortion of the $\mathrm{O}$ octahedra upon Fe substitution on

the B-site, i.e. by the local tetragonality defined as the ratio between the out-of-plane and in-plane $\mathrm{O}$-O distances $\left(\mathrm{O}_{\perp} / \mathrm{O}_{/ /}\right)$ of the $\mathrm{O}$ octahedron surrounding the $\mathrm{B}$ cation. In particular, they estimated by DFT calculations that the tetragonality of the outer sites changes depending on configuration. A sketch of four symmetrically-inequivalent configurations O1, I1, M2 and M3 - where "O" stands for outer, "I" for inner and "M" for mixed - and their local tetragonality profiles as obtained by DFT calculations ${ }^{22}$ are given in Figure $3 a$ and b, respectively.

The four selected configurations shown in Figure 3a are representative for the outer, mixed and inner groups, in which the two $\mathrm{Fe}$ dopants occupy respectively two, one or none of the perovskite unit cells adjacent to the $\mathrm{Bi}_{2} \mathrm{O}_{2}$ layers. In all these particular configurations, each of the two Fe dopants occupies a site on each adjacent column. Note that the lattice parameters and the tetragonal distortions are very similar for the configurations (as given in Birenbaum et al. ${ }^{22}$ ) belonging to the same group. As visible from the profiles in Figure $3 b$, the $\mathrm{I} 1$ and the $\mathrm{O} 1$ configurations differ for the tetragonality of the outer sites (corresponding to the B-site positions 1 and 4). On the other hand, the profiles of the mixed configurations are not symmetric, and an increase of $\mathrm{O}_{\perp} / \mathrm{O}_{/ /}$for the sites occupied by the Fe dopants is observed. Therefore, comparing the local tetragonality of $\mathrm{O}$ octahedra allows retrieving the local configuration of the Fe dopant atoms.

In order to experimentally investigate the preferential configuration of Fe dopant distribution, we performed a PeakPairs (PP) analysis by fitting the peaks corresponding to the A and $\mathrm{B}$ sites on the HAADF-STEM image and the peaks corresponding to the $\mathrm{O}$ columns on the inverted ABF-STEM image. The details about the fitting are given in the Material and Methods section. Figure 4a shows the ABF-STEM image of a portion of sample without OPBs. In Figure $4 \mathrm{~b}$ a map of the local tetragonality of the perovskite cells (c/a ratio) is shown, obtained as the ratio between the out-of-plane and inplane interatomic distances between $\mathrm{Bi}$ columns in the HAADF-STEM image. Additionally, the map of the local tetragonality of the octahedral sites $\left(\mathrm{O}_{\perp} / \mathrm{O}_{/ /}\right)$obtained from the corresponding ABF signal is presented in Figure 4c.

The results highlight that the perovskite cage of the $\mathrm{Bi}$ cations is mostly distorted in the outer cells next to the fluorite-like layers, which undergo a tetragonal distortion as large as 1.25. The profile of the tetragonal distortion of the perovskite cages shown in Figure 4d (black line) is symmetric for each of the 4 perovskite blocks, hence being not sensitive to the Fe dopant occupation sites. On the contrary, the profile for the tetragonality of the octahedral sites (purple line) shows strong asymmetries which can be related to the Fe occupation within the perovskite blocks.

To compare the experimental results with the calculated structures, a simulated ABF image of the M2 configuration is plotted superimposed to Figure $4 \mathrm{a}$ and marked by a white dashed box. The positions of the Fe dopants in the M2 configuration are marked by two asterisks next to the simulated portion of the image. Moreover, the $\mathrm{O}_{\perp} / \mathrm{O}_{/ /}$map and the corresponding tetragonal distortion profile for the simulated structure are also shown superimposed to Figure $4 \mathrm{c}$ and $\mathrm{d}$, respectively. The simulated map and the profile are in very good agreement with the experimental data for the lower perovskite block of Figure 4a. In particular, the data show that the highest tetragonal distortion of the $\mathrm{O}$ cage occurs when the 
Fe dopant atom occupies an outer perovskite site, giving rise to a tetragonal distortion $\mathrm{O}_{\perp} / \mathrm{O}_{/ /}$as large as 1.15 . Contrarily, a distortion of 1.03-1.05 is obtained when the $\mathrm{Fe}$ dopant occupies the inner site. This result confirms that the tetragonality of the octahedral site is highly sensitive to the dopant substitution.

On the base of our analysis, we can therefore label the Fe dopant distribution along the layered structure. The black arrows in Figure 4d mark the preferential planes in which the Fe dopant is localized in the analyzed portion of the specimen. Analyzing the experimental profile of the $\mathrm{O}$ octahedron tetragonality we are thus able to conclude that the $\mathrm{Fe}$ distribution within the perovskite blocks occurs giving rise to mixed configurations, i.e. one inner and one outer site are preferentially occupied. Localizing the sites showing a higher $\mathrm{O}$ octahedron tetragonality allows us for identifying the sites occupied by $\mathrm{Fe}$ dopants and thus retrieving the preferred mixed configuration.

The atomically-resolved EDX spectroscopy maps displayed in Figure 5 confirm these findings. In particular, the HAADFSTEM image of Figure 5a shows a portion of the Aurivillius sample containing three perovskite blocks. The elemental maps extracted from the acquired spectrum image for Bi-M $(2.4 \mathrm{kV})$, Ti-K $(4.5 \mathrm{kV})$ and $\mathrm{Fe}-\mathrm{K}(6.4 \mathrm{kV})$ edges are given in Figure 5b. Figure 5c shows the corresponding intensity profiles, obtained by integrating the elemental maps along their full width. The average preferential sites for Fe dopant can be seen as local maxima in the Fe-K edge profile. At these positions a decrease in the Ti-K edge profile is observed as expected for the substitutional doping of $\mathrm{Fe}$ on the B-site of the perovskite cell. The preferential positions have been marked by asterisks in the Figure $5 \mathrm{c}$ and correspond to a mixed configuration.

It must be noted that the ABF-STEM and EDX results shown here, demonstrating a preferential mixed configuration with the Fe cations distributed over inner and outer octahedral $\mathrm{B}$ sites, were acquired in very thin regions of the specimen. In these thin areas, typically below $30 \mathrm{~nm}$, the ABF-STEM signal shows directly interpretable image contrast for locating the oxygen columns. For thicker areas, however, an average over many inequivalent configurations along the sample thickness is expected as no preferential distribution of $\mathrm{Fe}$ can be observed by EDX mapping (see Figure S2, Supporting Information).

The study of the local ferroelectric properties of the Aurivillius phase is challenging, as the low symmetry polar phase displays complicated atomic displacements with respect to the paraelectric phase. As pointed out by Birenbaum et al. ${ }^{22}$ there exist three main symmetry modes that are involved in the transition from the paraelectric (exhibiting the $I 4 / \mathrm{mmm}$ symmetry) to the ferroelectric ( $A 2_{1} a m$ symmetry) phase of the 4-layered Aurivillius phase, i.e. the polar $\Gamma_{5}^{-}$mode and the two zone-boundary modes $X_{3}^{-}$and $X_{2}^{+}$. While the two zoneboundary modes only involve rotations and tilts of the oxygen octahedra, the polar $\Gamma_{5}^{-}$mode consists in an overall displacement of the ions in the $\mathrm{Bi}_{2} \mathrm{O}_{2}$ layers along the polar axis of the structure, i.e. along the in-plane [100] direction. Interestingly, this result is in close agreement with the case studies previously reported in literature for the two-layered Aurivillius system $\mathrm{SrBi}_{2} \mathrm{Ta}_{2} \mathrm{O}_{9} \cdot{ }^{45-47} \mathrm{In}$ particular, the rigid displacement of the $\mathrm{Bi}_{2} \mathrm{O}_{2}$ layers closely resembles the socalled "rigid layer mode" responsible for the polar instability in $\mathrm{SrBi}_{2} \mathrm{Ta}_{2} \mathrm{O}_{9}$.
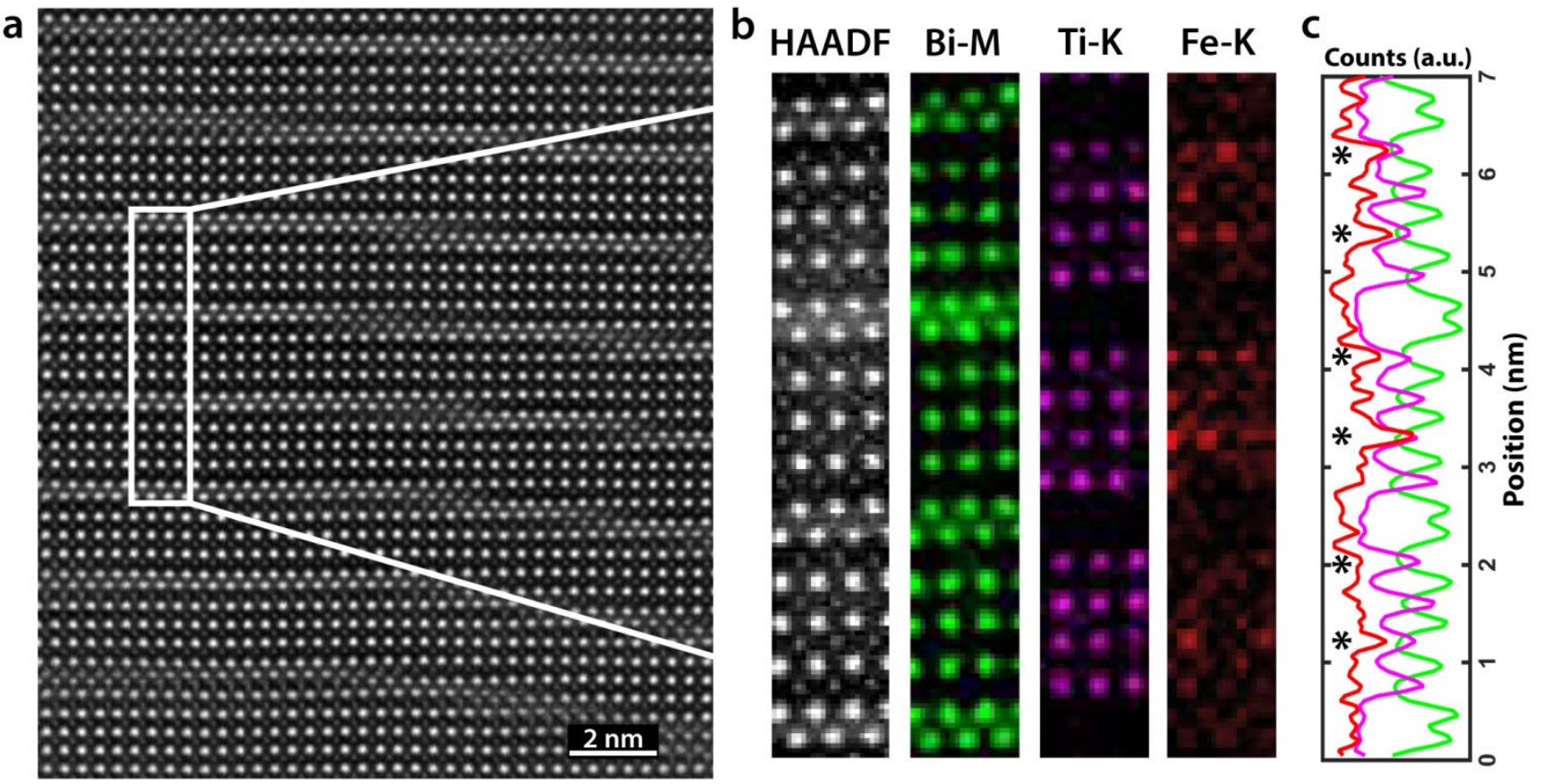

Figure 5. (a) High resolution HAADF-STEM of a portion of the Aurivillius thin film. The white box marks the region where the EDX spectrum image was acquired. (b) EDX elemental maps for Bi, Ti and Fe. (c) Color maps and intensity profiles of the Bi-M (green), Ti-K (blue) and Fe-K (red) signals along the film growing direction. The profiles demonstrate a preferential mixed configuration, where the preferential Fe dopant sites (marked by the asterisks) appear as maxima in the Fe-K profile and minima in the Ti-K profile. 

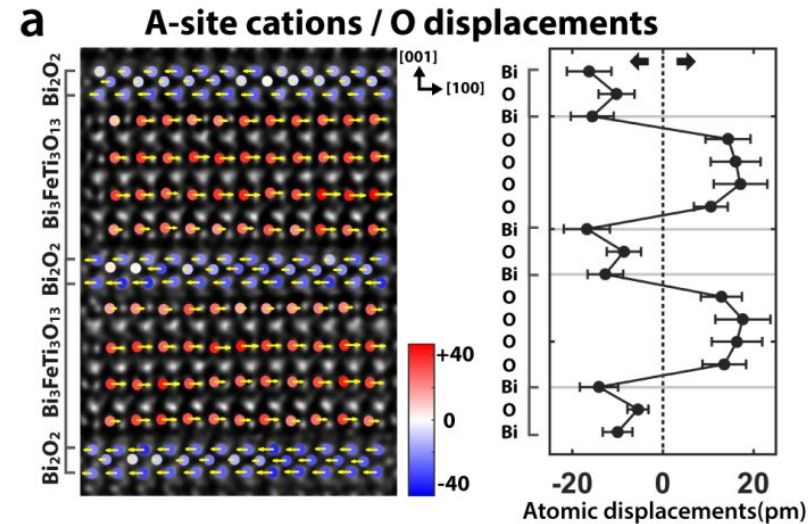

b
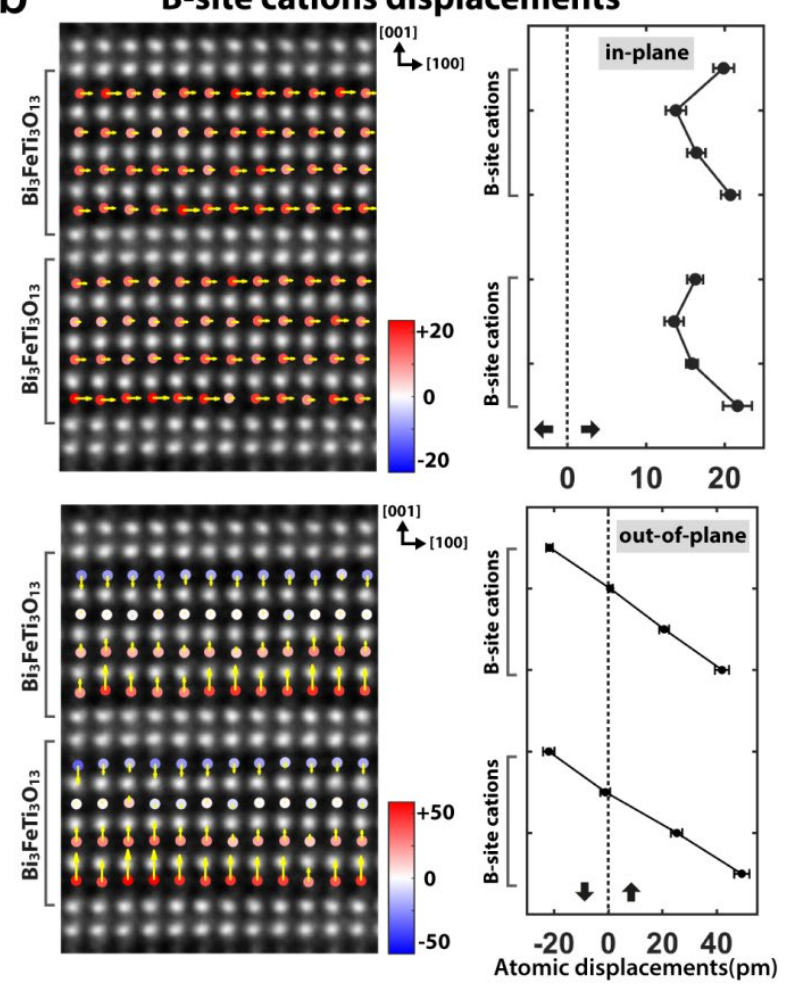

Figure 6. Quantitative analysis of (a) A-cations / O columns displacements and (b) B-site cations off-centering (both given in pm), carried out on inverted-ABF and HAADF signals respectively, in the [010] zone axis. The color polar plot and the yellow arrows give the magnitude and direction of the atomic displacements for the different sites in the $\mathrm{Bi}_{2} \mathrm{O}_{2}$ and $\mathrm{Bi}_{3} \mathrm{FeTi}_{3} \mathrm{O}_{13}$ layers.

From a detailed analysis of the symmetry modes in the 4layered Aurivillius phase, Birenbaum and coworkers ${ }^{22}$ observed that the polar $\Gamma_{5}^{-}$mode also involves a strong displacement of the B-site cations within the perovskite blocks, which is anti-parallel to the direction of the $\mathrm{Bi}_{2} \mathrm{O}_{2}$ shift, and a parallel shift of the $\mathrm{O}$ octahedra. These additional displacive instabilities appear to be similar to other A-site and B-site displacive modes in the perovskite block, which have been reported in different compounds, e.g. $\mathrm{Bi}_{2} \mathrm{WO}_{6}$ and $\mathrm{Bi}_{4} \mathrm{Ti}_{3} \mathrm{O}_{12}$.

The local ferroelectric properties of the BFTO thin film were analyzed by mapping the atomic-scale displacements from high-resolution HAADF/ABF-STEM signals. In Figure $6 \mathrm{a}$ we report the atomic scale investigation of the displacements induced by the $\Gamma_{5}^{-}$mode, visible as the simultaneous and anti-parallel shifts of the $\mathrm{Bi}_{2} \mathrm{O}_{2}$ layers and the $\mathrm{O}$ octahedra in the perovskite blocks. Starting from the experimental values of such displacements, it is possible to calculate the $\Gamma_{5}^{-}$mode amplitude. In particular, from the experimental displacements we obtained a mode amplitude of $147 \mathrm{pm}$, in excellent agreement with the theoretical value (140 pm) obtained from $a b$ initio calculation performed using PBEsol functional (a Perdew-Burke-Ernzerhof functional optimized for solid state systems). ${ }^{22}$ Interestingly, the polar $\Gamma_{5}$ mode also induces a remarkable off-centering of the B-site cations. Figure $6 \mathrm{~b}$ shows the in-plane and out-of-plane displacements of the cations in the B-site of the perovskite structure. A strong in-plane shift of the B cations proves that for the 4-layer Aurivillius phase a displacive instability is associated to the polar $\Gamma_{5}{ }^{-}$mode. As a direct consequence, for the analyzed system the conventional approach to polarization mapping by measuring the B-site off-centering from highresolution STEM images remains efficient and reliable for the investigated system.

The study of the structural distortions can be performed by comparing the experimental off-centering of the B-site cations with the ones of the theoretical mixed configuration, expected on the base of the previously reported ABF signal analysis and EDX mapping. Figure $7 \mathrm{a}$ and $7 \mathrm{c}$ show two images of the film along the [110] and [100] zone axes, respectively. For both orientations, the simulated HAADF-STEM signals (performed using the mixed configuration M2) are shown inside the white box. The polarization maps (yellow arrows) are plotted superimposed to the images. In Figure $7 \mathrm{~b}$ the profiles along the [001] direction of the atomic displacement components at the origin of the ferroelectric properties are given for both the experimental and simulated data. In the profiles, the displacement components are called respectively $\Delta \mathrm{x}$ and $\Delta \mathrm{y}$ for the in-plane and out-of-plane directions, as depicted in the sketch. The quantitative analysis of the displacements shows very good agreement between the experimental data and the simulation (black curve). In particular, $\Delta y$ - related to the outof-plane component of the polarization - has a zero net average value over the perovskite blocks, due to a compensation of the vertical atomic displacements for the even number of perovskite layers of the $m=4$ phase. A large displacement of the $\mathrm{B}$ cations in the outer perovskite sites along the c-axis of the Aurivillius structure has been reported by neutron diffraction experiments ${ }^{50}$ and, similarly to our observations, the opposite shifts of the B cations in the lower and upper perovskite blocks has been attributed to the vanishing polarization along the c-axis. ${ }^{51}$

On the contrary, the film displays a finite in-plane polarization arising from the net $\Delta x$ displacement of the $B$ cations, as large as of $\sim 25 \mathrm{pm}$. Such displacement corresponds to a polarization value of $\sim 50-55 \mu \mathrm{C} \mathrm{cm}^{-2}$ - obtained under the Born Effective Charge (BEC) approximation ${ }^{52,53}$ - in good agreement with the polarization value estimated for the mixed configuration of the BFTO by Birenbaum and coworkers. ${ }^{23}$ Notably, the in-plane displacement of the B cations at the origin of the ferroelectric polarization is anisotropic and occurs along the [100] direction of the Aurivillius structure corresponding to a [110]-type direction in the perovskite pseudocubic setting. In fact, taking the profile of the $\Delta x$ displacements along the [100] zone axis, no net in-plane displacements are detected (Figure 6d). The small asymmetries in the $\Delta y$ profile can be ascribed to small residual 
specimen mistilt, which are particularly relevant for the precise fitting of the atomic positions. These results confirm that the 4-layered Aurivillius system has an anisotropic ferroelectric behavior, being the polar axis along the [100] direction of the $A 2{ }_{1}$ am structure.

In order to investigate the ferroelectric domain structure in the single-crystalline Aurivillius thin films, we performed a combined DPC- and HAADF-STEM analysis. In medium resolution DPC-STEM, in fact, due to the lateral size of the slightly defocused probe extending over a few unit cells, the electric fields in the specimen plane induce a deflection of the transmitted beam that dominates over channeling effects. ${ }^{36} \mathrm{We}$ can therefore map the electric fields in the specimen plane by measuring the displacement of the transmitted convergent beam in the diffraction plane by means of a segmented detector (see Materials and Methods section for the experimental details). With respect to displacement mapping in high-resolution HAADF-STEM, medium resolution DPCSTEM allows the investigation of significantly larger portions of the specimen and the study of the ferroelectric domain structure in materials whose polarization exceed $10 \mu \mathrm{C} \mathrm{cm}^{-2}$. 37

Figure 8a shows an HAADF-STEM image over a large field of view of a BFTO thin film in cross-sectional geometry and Figure $8 \mathrm{~b}$ the results of the DPC-STEM analysis. By proper choice of the specimen orientation with respect to the detector geometry (see the scheme in Figure $8 \mathrm{~b}$ ), we can directly correlate the two differential signals (A-C) and (B-D) to the in-plane and out-of-plane components of the ferroelectric polarization. While no specific contrast arises in the DPC(AC) signal except for small fluctuations occurring within the perovskite blocks, faintly visible because of the low resolution of the de-magnified image, a clear contrast change can be observed in the DPC(B-D) signal for different portions along the c-axis of the film. In particular, the regions appearing with bright and dark contrast correspond to domains with opposite in-plane polarization vectors.

The boundary region at the interface between two planar domains was studied by polarization mapping from HAADFSTEM. Figure $8 \mathrm{c}$ shows a micrograph of a region containing a domain wall (DW). The polar map superimposed to the HAADF-STEM signal clearly demonstrates that the DW is located at the $\mathrm{Bi}_{2} \mathrm{O}_{2}$ layer and separates two regions in which the film has opposite in-plane polarizations. The opposite polarization vectors in the two ferroelectric domains arises from an opposite displacement of the B-site cations, which can occur along the [1-10] or [-110] directions as shown in Figure $8 \mathrm{~d}$.

The effect of OPBs on the nanoscale ferroelectric properties can be finally elucidated by performing a polarization analysis across a region showing this structural defect. Figure 8e shows an HAADF-STEM image superimposed on a polarization map across a region containing several OPBs, whereas Figure $8 \mathrm{f}$ shows the maps of the atomic displacement components. The polarization of the structure appears to be only slightly perturbed by the defects, due to the occurrence of small regions in which $m=3$ or 5 in the vicinity of the OPBs. Interestingly, our STEM results prove that the occurrence of in-plane ferroelectric domains is not related to defects, like e.g. OPBs, which has been proved to have a beneficial effect on the fatigue properties of the materials. ${ }^{14,54}$

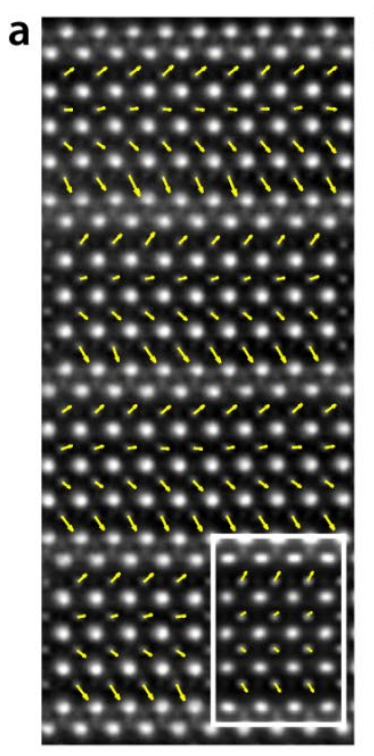

b
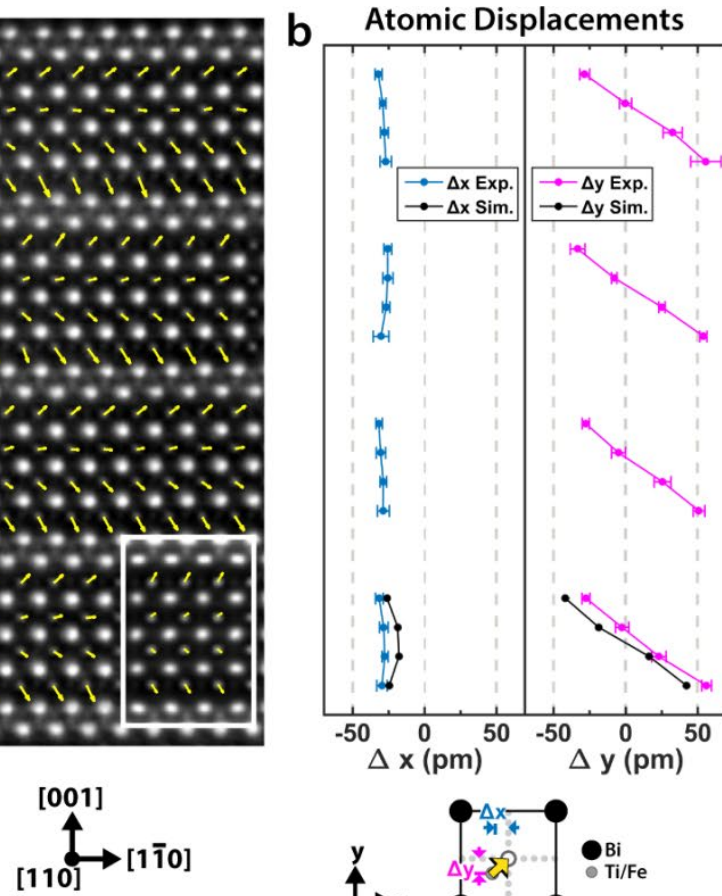

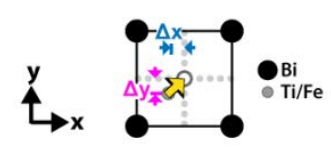

C

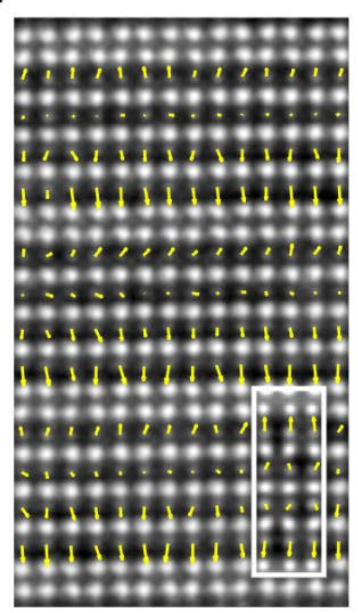

d
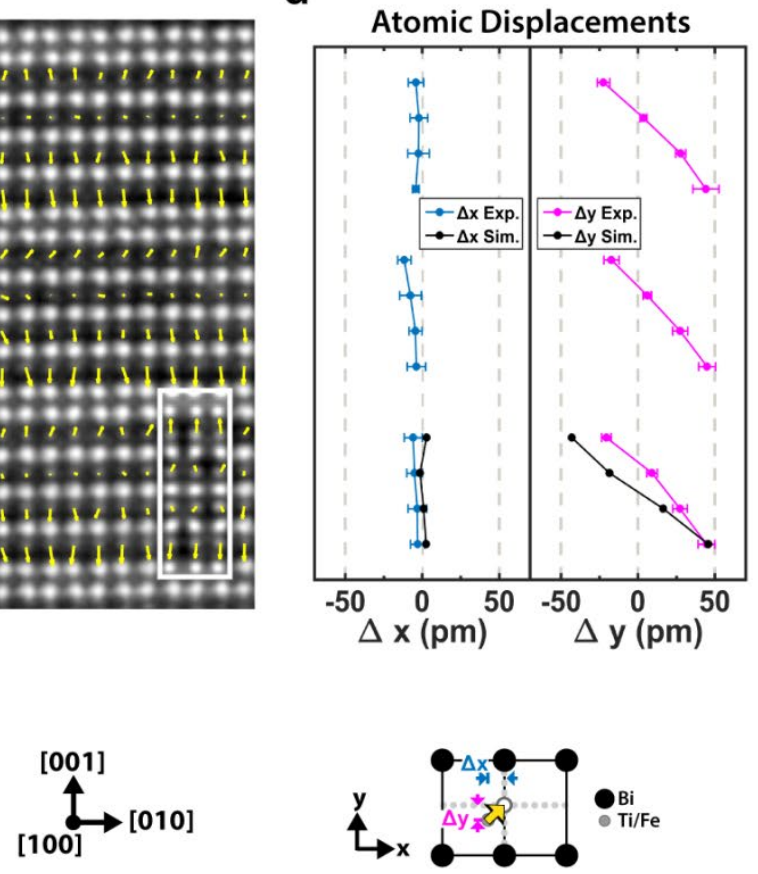

Figure 7. (a, c) Polarization maps, as obtained from the off-centering of the B-site cations, superimposed to HAADF-STEM images along the [110] and [100] zones axes, respectively. The corresponding simulated signals in both zone axes (generated using the M2 configuration) are given inside the white boxes. (b, d) Profiles along the [001] direction of the atomic displacement of the B cations for the two different zone axes, respectively. The profiles of the simulated signals are plotted in black. The displacement geometry in the two different orientations of the perovskite system is given in the sketches. 

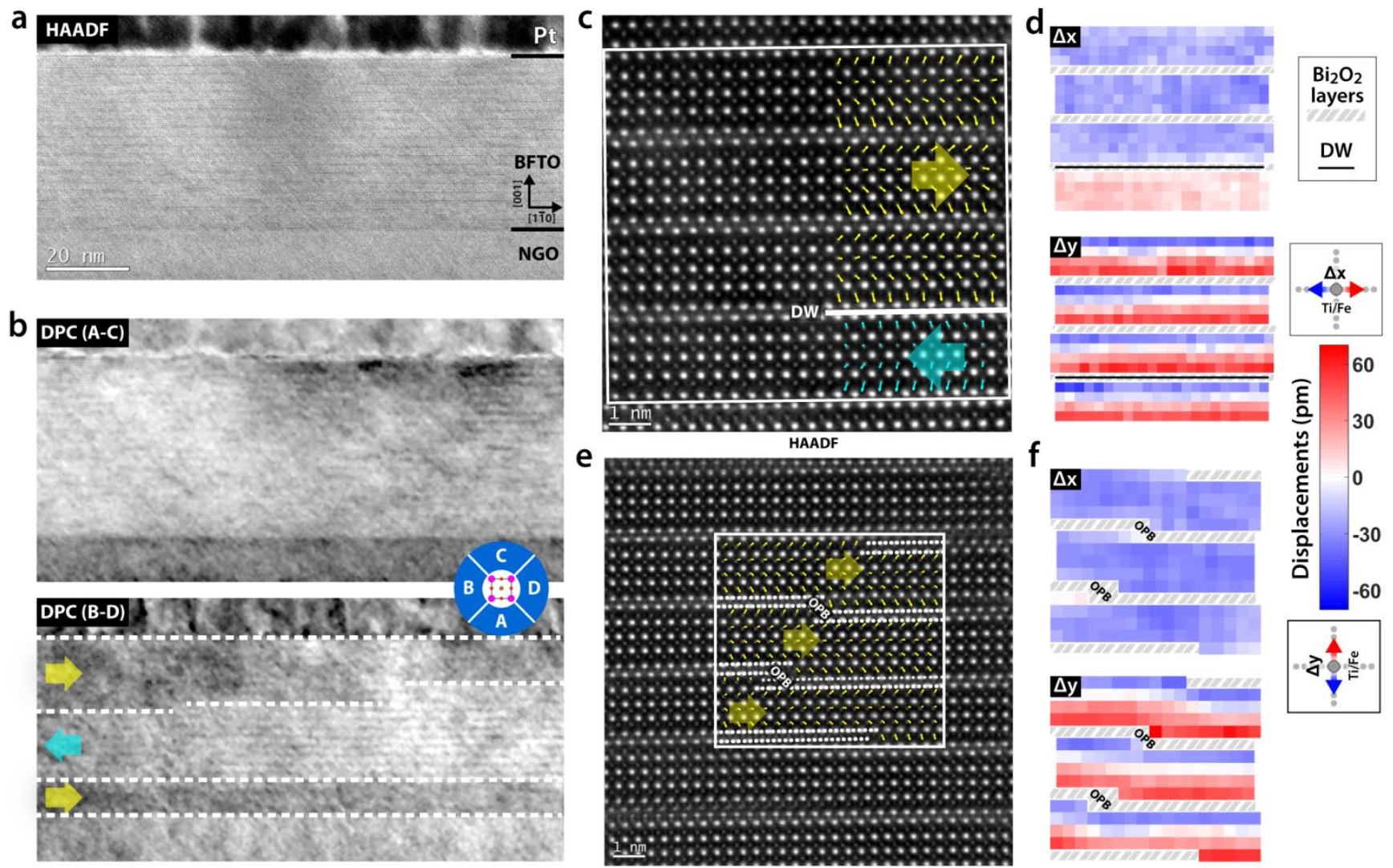

Figure 8. (a) HAADF-STEM image of a large portion of the BFTO film. (b) Corresponding DPC-STEM signals, which are related to the in-plane (D-B) and out-of-plane (A-C) components of the electric field. c) High resolution HAADF-STEM image over a portion of the sample containing a DW. As visible from the arrows, the domain wall is located at the $\mathrm{Bi}_{2} \mathrm{O}_{2}$ layer. A map of the in-plane and out-of-plane displacements for the area marked by the white-dashed box in (c) is given in (d). (e) HAADF-STEM image and polarization map in the proximity of a OPBs and (f) corresponding displacement maps for the B-site cations.

\section{CONCLUSIONS}

In our work, we demonstrated the realization of high-quality single-crystalline $\mathrm{Bi}_{5} \mathrm{FeTi}_{3} \mathrm{O}_{15}$ Aurivillius thin films epitaxially grown on orthorhombic $\mathrm{NdGaO}_{3}$ substrate by PLD. The grown films are single-crystalline and completely c-oriented.

The presented structural investigations demonstrate that the films grow in a strain-relaxed state, which occurs due to the formation of structural defects - like e.g. OPBs. Due to the relaxed state of the system, we found that the distribution of Fe dopants gives rise to mixed configurations, i.e. contemporary $\mathrm{Fe}$ substitution of one inner and one outer perovskite site in the Aurivillius blocks. Because of the presence of numerous OPBs, even selecting a substrate with low epitaxial mismatch (as it is the case for NGO), it is not possible to induce a strain state in the Aurivillius phase. These findings suggest that controlling the Fe dopant configuration by epitaxial strain is not feasible in real Aurivillius systems.

The analysis of simultaneously acquired HAADF-/ABFSTEM signals allows us to conclude that the tetragonality of the $\mathrm{O}$ octahedra $\left(\mathrm{O}_{\perp} / \mathrm{O}_{/ /}\right)$is more sensitive to $\mathrm{B}$ cation substitution than the distortion of the perovskite cell (c/a). The values of the $\mathrm{O}$ octahedra tetragonality are in good agreement with the DFT calculations, with a maximum value for the outer perovskite sites occupied by Fe. More generally, our results prove that the $\mathrm{O}$ octahedra tetragonality ratio estimated by fitting the positions of $\mathrm{O}$ atomic columns in the ABF signal can be successfully employed as an efficient tool to identify the sites preferentially occupied by the Fe dopants in the layered Aurivillius phase. This method represents a convenient approach, which can be eventually combined with EDX mapping, to reveal the dopant distribution in doped layered oxides.

The polarization analysis of BFTO films shows an anisotropic ferroelectric behavior, with a complex pattern of atomic displacements originated by the polar $\Gamma_{5}^{-}$mode. In addition to the rigid shift of the $\mathrm{Bi}_{2} \mathrm{O}_{2}$ layers, such mode induces a displacive off-centering of the $\mathrm{B}$-site cations along the [100] direction of the Aurivillius structure. The displacements of the B-site cations can be used to map the ferroelectric polarization, as it is commonly used for ferroelectric oxides. The estimated local ferroelectric polarization $\left.(\sim 50-55 \mu \mathrm{C} \mathrm{cm})^{-2}\right)$ for the structure with $A 2{ }_{1} a m$ symmetry is in excellent agreement with the value predicted by theory and appears to be very robust against doping. Interestingly, buried in-plane ferroelectric domains are experimentally observed. The existence of such in-plane ferroelectric domains has been proven by means of DPCSTEM and displacement analysis in high-resolution HAADFSTEM. In particular, DPC-STEM is particularly promising for the study of buried polar structures whose polarization is not directly accessible by surface-sensitive techniques such as piezoresponse force microscopy ${ }^{55,56}$ (PFM). 
Furthermore, the domain walls between areas of the film with opposite ferroelectric polarizations lie in the $\mathrm{Bi}_{2} \mathrm{O}_{2}$ layers separating the perovskite blocks and they are not pinned to structural defects. On the other hand, OPBs do not affect the microscopic ferroelectric polarization, thus not having any detrimental effect on the polar properties of the thin film.

The observed planar domains, here reported for 4-layer Aurivillius thin films, can play an important role in the macroscopic ferroelectric behavior of the system. Such buried polar domains have to be taken into account in order to properly describe the material properties and might be decisive for the implementation of thin films of layered perovskite systems - like e.g. Aurivillius phases - into ferroelectric devices.

\section{ASSOCIATED CONTENT}

\section{Supporting Information}

This material is available free of charge via the Internet at http://pubs.acs.org.

Thin film strain analysis by Geometric Phase Analysis, Additional EDXS analysis (PDF)

\section{AUTHOR INFORMATION}

\section{Corresponding Author}

* Email: marco.campanini@empa.ch.

\section{Author Contributions}

M.T. grew the thin films and performed the XRD/AFM investigations. M.C., R.E. and M.D.R. carried out the STEM experiments and M.C. performed the data analysis. C.E. provided the structural files obtained from DFT calculation and discussed the comparison between experiments and simulations. M.C. wrote the manuscript. M.D.R. and M.T. coordinated the project. All authors have given approval to the final version of the manuscript.

\section{Notes}

The authors declare no competing financial interest.

\section{ACKNOWLEDGMENTS}

This research was supported by SNSF project no. 200021_175926. M.C. and M.D.R. thanks NCCR-MARVEL project funded by the Swiss National Science Foundation (SNSF) for the financial support.

\section{REFERENCES}

(1) Choi, K. J.; Biegalski, M.; Li, Y. L.; Sharan, A.; Schubert, J.; Uecker, R.; Reiche, P.; Chen, Y. B.; Pan, X. Q.; Gopalan, V.; et al. Enhancement of Ferroelectricity in Strained BaTiO3 Thin Films. Science (80-. ). 2004, 306 (5698), 1005-1009.

(2) Zhang, J. X.; He, Q.; Trassin, M.; Luo, W.; Yi, D.; Rossell, M. D.; Yu, P.; You, L.; Wang, C. H.; Kuo, C. Y.; et al. Microscopic Origin of the Giant Ferroelectric Polarization in Tetragonal-like BiFeO 3. Phys. Rev. Lett. 2011, 107 (14), 147602.

(3) Ederer, C.; Spaldin, N. A. Effect of Epitaxial Strain on the Spontaneous Polarization of Thin Film Ferroelectrics. Phys. Rev. Lett. 2005, 95 (25), 257601.

(4) Haeni, J. H.; Irvin, P.; Chang, W.; Uecker, R.; Reiche, P.; Li, Y. L.; Choudhury, S.; Tian, W.; Hawley, M. E.; Craigo, B.; et al. Room-Temperature Ferroelectricity in Strained SrTiO3. Nature 2004, 430 (7001), 758-761.

(5) De Luca, G.; Strkalj, N.; Manz, S.; Bouillet, C.; Fiebig, M.; Trassin, M. Nanoscale Design of Polarization in Ultrathin Ferroelectric Heterostructures. Nat. Commun. 2017, 8 (1), 1419.
Doping BiFeO3: Approaches and Enhanced Functionality. Phys. Chem. Chem. Phys. 2012, 14 (46), 15953-15962.

(7) Lee, Y.-H.; Wu, J.-M.; Lai, C.-H. Influence of La Doping in Multiferroic Properties of BiFeO3 Thin Films. Appl. Phys. Lett. 2006, 88 (4), 042903.

Cao, G.; Chikara, S.; Lin, X. N.; Elhami, E.; Durairaj, V.; Schlottmann, P. Itinerant Ferromagnetism to Insulating Antiferromagnetism: A Magnetic and Transport Study of Single Crystal $\mathrm{SrRu} 1-\mathrm{x} \quad \mathrm{Mn} \mathrm{x} \quad \mathrm{O} 3 \quad(0 \square \mathrm{x} \& \mathrm{lt} ; 0.60)$. Phys. Rev. B 2005, 71 (3), 035104

Zurbuchen, M. A.; Freitas, R. S.; Wilson, M. J.; Schiffer, P.; Roeckerath, M.; Schubert, J.; Biegalski, M. D.; Mehta, G. H.; Comstock, D. J.; Lee, J. H.; et al. Synthesis and Characterization of an $\mathrm{N}=6$ Aurivillius Phase Incorporating Magnetically Active Manganese, Bi7(Mn,Ti)6O21. Appl. Phys. Lett. 2007, 91 (3), 033113.

(10) Mundy, J. A.; Brooks, C. M.; Holtz, M. E.; Moyer, J. A.; Das, H.; Rébola, A. F.; Heron, J. T.; Clarkson, J. D.; Disseler, S. M.; Liu, Z.; et al. Atomically Engineered Ferroic Layers Yield a Room-Temperature Magnetoelectric Multiferroic. Nature 2016, 537 (7621), 523-527.

(11) Liu, S.; Miiller, W.; Liu, Y.; Avdeev, M.; Ling, C. D. SillenAurivillius Intergrowth Phases as Templates for Naturally Layered Multiferroics. Chem. Mater. 2012, 24 (20), 3932-3942.

Ruddlesden, S. N.; Popper, P.; IUCr. The Compound $\mathrm{Sr}_{3} \mathrm{Ti}_{2} \mathrm{O}$ 7 and Its Structure. Acta Crystallogr. 1958, 11 (1), 54-55.

Aurivillius, B. Mixed Bismuth Oxides with Layer Lattices. II Structure of Bi4 Ti3 O12. Ark. foer Kemi 1949, 1, 499-512.

de Araujo, C. A.-P.; Cuchiaro, J. D.; McMillan, L. D.; Scott, M. C.; Scott, J. F.; Mc Millan, D. L.; Scott, M. C.; Scott, J. F.; McMillan, L. D.; Scott, M. C.; et al. Fatigue-Free Ferroelectric Capacitors with Platinum Electrodes. Nature 1995, 374 (6523), 627-629.

(15) Sivakumar, T.; Itoh, M. New Ferroelectric Aurivillius Oxides: Incorporation of $\mathrm{Sc}^{3+}$ in Stoichiometric Compositions. Chem. Mater. 2011, 23 (2), 129-131.

(16) Surta, T. W.; Manjón-Sanz, A.; Qian, E. K.; Mansergh, R. H.; Tran, T. T.; Fullmer, L. B.; Dolgos, M. R. Dielectric and Ferroelectric Properties in Highly Substituted $\mathrm{Bi}_{2} \mathrm{Sr}(\mathrm{A}) \mathrm{TiNb}_{2} \mathrm{O}_{12}$ (A $\left.=\mathrm{Ca}^{2+}, \mathrm{Sr}^{2+}, \mathrm{Ba}^{2+}\right)$ Aurivillius Phases. Chem. Mater. 2017, 29 (18), 7774-7784

(17) Chen, X.; Xiao, J.; Xue, Y.; Zeng, X.; Yang, F.; Su, P. Room Temperature Multiferroic Properties of Ni-Doped Aurivillus Phase Bi5Ti3FeO15. Ceram. Int. 2014, 40 (2), 2635-2639.

(18) Keeney, L.; Downing, C.; Schmidt, M.; Pemble, M. E. Nicolosi, V.; Whatmore, R. W. Direct Atomic Scale Determination of Magnetic Ion Partition in a Room Temperature Multiferroic Material. Sci. Rep. 2017, 7 (1), 1737.

(19) Mao, X.; Wang, W.; Chen, X.; Lu, Y. Multiferroic Properties of Layer-Structured Bi5Fe0.5Co0.5Ti3O15 Ceramics. Appl. Phys. Lett. 2009, 95 (8), 082901.

(20) Wang, G.; Chen, Z.; He, H.; Meng, D.; Yang, H.; Mao, X.; Pan, Q.; Chu, B.; Zuo, M.; Sun, Z.; et al. Room Temperature Exchange Bias in Structure-Modulated Single-Phase Multiferroic Materials. Chem. Mater. 2018, 30 (17), 6156-6163.

Park, B. H.; Kang, B. S.; Bu, S. D.; Noh, T. W.; Lee, J.; Jo, W. Lanthanum-Substituted Bismuth Titanate for Use in NonVolatile Memories. Nature 1999, 401 (6754), 682-684.

(22) Birenbaum, A. Y.; Ederer, C. Potentially Multiferroic Aurivillius Phase Bi5FeTi3 O15: Cation Site Preference, Electric Polarization, and Magnetic Coupling from First Principles. Phys. Rev. B - Condens. Matter Mater. Phys. 2014, 90 (21), 214109.

(23) Birenbaum, A. Y.; Ederer, C. Controlling the Cation Distribution and Electric Polarization with Epitaxial Strain in Aurivillius-Phase Bi5FeTi3O15. Appl. Phys. Lett. 2016, 108 (8), 82903.

(24) Mao, X.; Wang, W.; Chen, X.; Lu, Y. Multiferroic Properties of Layer-Structured Bi5Fe0.5 Co0.5 Ti3O15 Ceramics. Appl. Phys. Lett. 2009, 95 (8), 82901.

(25) Keeney, L.; Maity, T.; Schmidt, M.; Amann, A.; Deepak, N.; Petkov, N.; Roy, S.; Pemble, M. E.; Whatmore, R. W. Magnetic Field-Induced Ferroelectric Switching in Multiferroic Aurivillius Phase Thin Films at Room Temperature. J. Am. Ceram. Soc. 2013, 96 (8), 2339-2357.

(26) Jartych, E.; Pikula, T.; Mazurek, M.; Lisinska-Czekaj, A.; 
Czekaj, D.; Gaska, K.; Przewoznik, J.; Kapusta, C.; Surowiec Z. Antiferromagnetic Spin Glass-like Behavior in Sintered Multiferroic Aurivillius Bim $+1 \mathrm{Ti} 3 \mathrm{Fem}-3 \mathrm{O} 3 \mathrm{~m}+3$ Compounds. $J$ Magn. Magn. Mater. 2013, 342, 27-34.

(27) Faraz, A.; Maity, T.; Schmidt, M.; Deepak, N.; Roy, S.; Pemble, M. E.; Whatmore, R. W.; Keeney, L. Direct Visualization of Magnetic-Field-Induced Magnetoelectric Switching in Multiferroic Aurivillius Phase Thin Films. J. Am. Ceram. Soc. 2017, 100 (3), 975-987.

(28) Li, Z.; Ma, J.; Gao, Z.; Viola, G.; Koval, V.; Mahajan, A.; Li, X.; Jia, C.; Nan, C.; Yan, H. Room Temperature Magnetoelectric Coupling in Intrinsic Multiferroic Aurivillius Phase Textured Ceramics. Dalt. Trans. 2016, 45 (36), 14049-14052.

(29) Liu, J.; Bai, W.; Yang, J.; Xu, W.; Zhang, Y.; Lin, T.; Meng, X.; Duan, C.-G.; Tang, X.; Chu, J. The Cr-Substitution Concentration Dependence of the Structural, Electric and Magnetic Behaviors for Aurivillius $\mathrm{Bi}_{5} \mathrm{Ti}_{3} \mathrm{FeO}_{15}$ Multiferroic Ceramics. J. Appl. Phys. 2013, 114 (23), 234101.

(30) Yu, Z.; Yu, B.; Liu, Y.; Zhou, P.; Jiang, J.; Liang, K.; Lu, Y.; Sun, H.; Chen, X.; Ma, Z.; et al. Enhancement of Multiferroic Properties of Aurivillius Bi5Ti3FeO15 Ceramics by Co Doping. Ceram. Int. 2017, 43 (17), 14996-15001.

(31) Lee, H. N.; Hesse, D.; Zakharov, N.; Gösele, U. Ferroelectric Bi3.25La0.75Ti3O12 Films of Uniform a-Axis Orientation on Silicon Substrates. Science 2002, 296 (5575), 2006-2009.

(32) Pignolet, A.; Schäfer, C.; Satyalakshmi, K. M.; Harnagea, C. Hesse, D.; Gösele, U. Orientation Dependence of Ferroelectricity in Pulsed-Laser-Deposited Epitaxial Bismuth-Layered Perovskite Thin Films. Appl. Phys. A Mater. Sci. Process. 2000, 70 (3), 283-291.

(33) Zhang, P. F.; Deepak, N.; Keeney, L.; Pemble, M. E.; Whatmore, R. W. The Structural and Piezoresponse Properties of CAxis-Oriented Aurivillius Phase Bi 5 Ti 3 FeO 15 Thin Films Deposited by Atomic Vapor Deposition. Cite as Appl. Phys. Lett 2012, 101, 112903

(34) Findlay, S. D.; Shibata, N.; Sawada, H.; Okunishi, E.; Kondo, Y.; Ikuhara, Y. Dynamics of Annular Bright Field Imaging in Scanning Transmission Electron Microscopy. Ultramicroscopy 2010, 110 (7), 903-923.

(35) Shibata, N.; Findlay, S. D.; Kohno, Y.; Sawada, H.; Kondo, Y.; Ikuhara, Y. Differential Phase-Contrast Microscopy at Atomic Resolution. Nat. Phys. 2012, 8, 611 .

(36) Lubk, A.; Zweck, J. Differential Phase Contrast: An Integral Perspective. Phys. Rev. A 2015, 91 (2), 23805.

(37) Campanini, M.; Erni, R.; Yang, C.; Ramesh, R.; Rossell, M. D. Periodic Giant Polarization Gradients in Doped BiFeO3 Thin Films. Nano Lett. 2018, 18 (2), 717-724.

(38) Grillo, V.; Rotunno, E. STEM_CELL A Software Tool for Electron Microscopy Part I- $\overline{\text { Simulations. Ultramicroscopy }}$ 2013, 125 (C), 97-111.

(39) Jones, L.; Yang, H.; Pennycook, T. J.; Marshall, M. S. J.; Van Aert, S.; Browning, N. D.; Castell, M. R.; Nellist, P. D. Smart Align-a New Tool for Robust Non-Rigid Registration of Scanning Microscope Data. Adv. Struct. Chem. Imaging 2015, 1 (1), 8 .

(40) Du, H. A Nonlinear Filtering Algorithm for Denoising HR(S)TEM Micrographs. Ultramicroscopy 2015, 151, 62-67.

(41) Yankovich, A. B.; Berkels, B.; Dahmen, W.; Binev, P.; Sanchez S. I.; Bradley, S. A.; Li, A.; Szlufarska, I.; Voyles, P. M. Picometre-Precision Analysis of Scanning Transmission Electron Microscopy Images of Platinum Nanocatalysts. Nat. Commun. 2014, 5 (1), 4155 .

(42) Hervoches, C. H.; Snedden, A.; Riggs, R.; Kilcoyne, S. H.; Manuel, P.; Lightfoot, P. Structural Behavior of the Four-Layer Aurivillius-Phase Ferroelectrics SrBi4Ti4O15 and Bi5Ti3FeO15. J. Solid State Chem. 2002, 164 (2), 280-291.

(43) He, J. Q.; Vasco, E.; Dittmann, R.; Wang, R. H. Growth Dynamics and Strain Relaxation Mechanisms in BaTiO3 Pulsed Laser Deposited on SrRuO3 SrTiO3. Phys. Rev. B - Condens. Matter Mater. Phys. 2006, 73 (12), 125413.

(44) Momma, K.; Izumi, F.; IUCr. VESTA 3 for Three-Dimensional Visualization of Crystal, Volumetric and Morphology Data. J. Appl. Crystallogr. 2011, 44 (6), 1272-1276.

(45) Shimakawa, Y.; Kubo, Y.; Nakagawa, Y.; Goto, S.; Kamiyama T.; Asano, H.; Izumi, F. Crystal Structure and Ferroelectric
Properties of $\mathrm{A}$ Bi 2 Ta 2 O $9(\mathrm{~A}=\mathrm{Ca}, \mathrm{Sr}$, and $\mathrm{Ba})$. Phys. Rev. B 2000, 61 (10), 6559-6564.

(46) Perez-Mato, J. M.; Aroyo, M.; García, A.; Blaha, P.; Schwarz, K.; Schweifer, J.; Parlinski, K. Competing Structural Instabilities in the Ferroelectric Aurivillius Compound $\mathrm{Sr}$ Bi 2 Ta 2 O 9. Phys. Rev. B 2004, 70 (21), 214111.

(47) Stachiotti, M. G.; Rodriguez, C. O.; Ambrosch-Draxl, C.; Christensen, N. E. Electronic Structure and Ferroelectricity in SrBi 2 Ta 2 O 9. Phys. Rev. B 2000, 61 (21), 14434-14439.

(48) Machado, R.; Stachiotti, M. G.; Migoni, R. L.; Tera, A. H. FirstPrinciples Determination of Ferroelectric Instabilities in Aurivillius Compounds. Phys. Rev. B 2004, 70 (21), 214112.

(49) Perez-Mato, J. M.; Blaha, P.; Schwarz, K.; Aroyo, M.; Orobengoa, D.; Etxebarria, I.; García, A. Multiple Instabilities in Bi 4 Ti 3 O 12 : A Ferroelectric beyond the Soft-Mode Paradigm. Phys. Rev. B 2008, 77 (18), 184104.

(50) and, C. H. H.; Lightfoot*, P. A Variable-Temperature Powder Neutron Diffraction Study of Ferroelectric Bi4Ti3O12. 1999.

(51) Shrinagar, A.; Garg, A.; Prasad, R.; Auluck, S.; IUCr. Phase Stability in Ferroelectric Bismuth Titanate: A First-Principles Study. Acta Crystallogr. Sect. A Found. Crystallogr. 2008, 64 (3), 368-375.

(52) King-Smith, R. D.; Vanderbilt, D. Theory of Polarization of Crystalline Solids. Phys. Rev. B 1993, 47 (3), 1651-1654.

(53) Resta, R. Macroscopic Polarization in Crystalline Dielectrics: The Geometric Phase Approach. Rev. Mod. Phys. 1994, 66 (3), 899-915.

(54) Anderson, J. S.; Hutchison, J. L. The Study of Long Range Order in Hexagonal Barium Ferrite Layer Structures. Contemp. Phys. 1975, 16 (5), 443-467.

(55) Güthner, P.; Dransfeld, K. Local Poling of Ferroelectric Polymers by Scanning Force Microscopy. Appl. Phys. Lett. 1992, 61 (9), 1137-1139.

(56) Kalinin, S. V.; Rodriguez, B. J.; Kim, S.-H.; Hong, S.-K.; Gruverman, A.; Eliseev, E. A. Imaging Mechanism of Piezoresponse Force Microscopy in Capacitor Structures. Appl. Phys. Lett. 2008, 92 (15), 152906 
Table of Contents

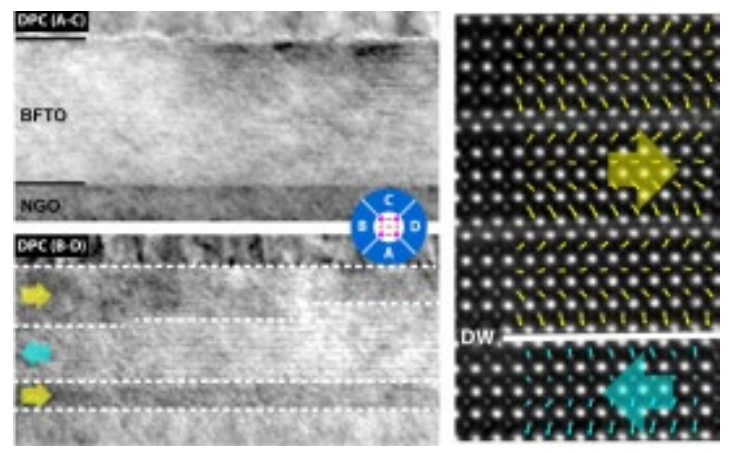

